\title{
Quantum Electrical Dipole in Triangular Systems: a Model for Spontaneous Polarity in Metal Clusters
}

\author{
Philip B. Allen ${ }^{1,2}$, Alexander G. Abanov ${ }^{1}$, and Ryan Requist ${ }^{1}$ \\ ${ }^{1}$ Department of Physics and Astronomy, Stony Brook University, \\ Stony Brook, NY 11794-3800, U.S.A. \\ ${ }^{2}$ Department of Applied Physics and Applied Mathematics \\ Columbia University, New York, NY 10032, U.S.A.
}

(Dated: November 20, 2018)

\begin{abstract}
Triangular symmetric molecules with mirror symmetry perpendicular to the 3 -fold axis are forbidden to have a fixed electrical dipole moment. However, if the ground state is orbitally degenerate and lacks inversion symmetry, then a "quantum" dipole moment does exist. The system of 3 electrons in $\mathrm{D}_{3 h}$ symmetry is our example. This system is realized in triatomic molecules like $\mathrm{Na}_{3}$. Unlike the fixed dipole of a molecule like water, the quantum moment does not point in a fixed direction, but lies in the plane of the molecule and takes quantized values $\pm \mu_{0}$ along any direction of measurement in the plane. An electric field $\vec{F}$ in the plane leads to a linear Stark splitting $\pm \mu_{0}|\vec{F}|$. We introduce a toy model to study the effect of Jahn-Teller distortions on the quantum dipole moment. We find that the quantum dipole property survives when the dynamic Jahn-Teller effect is included, if the distortion of the molecule is small. Linear Stark splittings are suppressed in low fields by molecular rotation, just as the linear Stark shift of water is suppressed, but will be revealed in moderately large applied fields and low temperatures. Coulomb correlations also give a partial suppression.
\end{abstract}

\section{INTRODUCTION}

Moro et al. (de Heer's group [1, 2] ) found an unexpected low temperature electrical dipole, apparently not classical, in nearly all clusters of $N$ niobium atoms with $2<N<150$. Here we use the $\mathrm{X}_{3}$ molecule to propose an answer to the question, what a non-classical electrical dipole might be. Our model electrical dipole bears a close analogy to the non-classical magnetic moment of a spin $1 / 2$ particle. It is meaningless to ask the direction of the moment of an isolated electron, because different components of the moment operator do not commute. In a field $\vec{B}$ the degeneracy with respect to spin degrees of freedom is lifted and the ground state has energy $E_{0}=-\mu_{B}|\vec{B}|$ and is characterized by having maximal projection of the moment $\vec{\mu}_{M}=\left\langle 0\left|\hat{\vec{\mu}}_{M}\right| 0\right\rangle=\mu_{B} \hat{B}$ along the magnetic field.

If the electron is not isolated but in contact with a heat bath, the statistical average of the moment is $\vec{\mu}_{M}=\mu_{B} \tanh \left(\mu_{B}|\vec{B}| / k_{B} T\right) \hat{B}$. The susceptibility has the Curie form $\chi=\mu_{B}^{2} / k_{B} T$. The thermal behavior at weak applied fields is classical [3], with a well-defined linear response $\vec{\mu}_{M}=\chi \vec{B}$. The operational definition of a dipole is a Curie response of the isolated system, and the moment is found from $\sqrt{\chi T}$. Classically we know that the moment points at some angle $\theta$ to the field and precesses. Quantum mechanics forces a more abstract view, which replaces the direction of the moment by the "quantization axis."

Usually we think of an electrical dipole moment $\vec{\mu}$ on a molecule in classical terms. When rigidly fixed in space, the moment has a value which is not quantized in atomic units $e a_{B}$. Its value is an accidental result of chemistry. In the frame of the molecule, the components of the electric dipole moment operator commute, so a direction can be defined in this frame. A thermalized vapor of molecules has moments randomly oriented. In an electric field $\vec{F}$ there is a net polarization proportional to $\vec{F}$, corresponding to an average dipole per molecule $\chi \vec{F}$, with the Curie form $\chi=\mu^{2} / 3 k_{B} T$ [3]. Quantum mechanics enters in the usual picture when $k_{B} T$ is small enough that the spacing of quantized rotational levels $E_{\mathrm{rot}}=\left(\hbar^{2} / 2 I\right) L(L+1)$ is no longer negligible. The ground state $L=0$ has full rotational symmetry with zero average dipole, and the Curie divergence is cut off, replaced by $\chi \rightarrow \mu^{2} /\left(\hbar^{2} / 2 I\right)[\underline{3}, 4]$.

More interesting quantum effects, including noncommutation of the relevant matrix elements of the electric dipole operator, may occur when the molecule has an orbitally degenerate ground state, and lacks an inversion center. We consider the simplest case, with equilateral triangular $\left(\mathrm{D}_{3 h}\right)$ symmetry. A fixed (classical) electrical dipole is inconsistent with $\mathrm{D}_{3 h}$ symmetry. To quote Townes and Schawlow, "there can be no dipole moment perpendicular to the axis of a symmetric top." [5]. This statement is also true in quantum mechanics if the ground state is non-degenerate. However, there is a nonzero electrical dipole matrix in the doubly degenerate $E$ representation of $\mathrm{D}_{3 h}$. This matrix has non-commuting Cartesian components. The moment exhibits quantum behavior similar to the spin of an electron. Further quantum effects suppress this moment, similar to the way zero-point rotation suppresses the usual molecular moment $[3,[4,6]$, but there are very interesting differences. Orbital magnetic moments also occur in this model, and are discussed in Appendix A

Our model (Sec. III) is a simplified picture of an alkali trimer like $\mathrm{Na}_{3}$. We start with a rigid equilateral triangle geometry, fixed in space, representing, for example, three atoms. All electrons except the outer 3 valence electrons (one per atom) are frozen. One alternate realization is the "R-center" in an alkali halide crystal [7]. In crys- 
talline $\mathrm{NaF}$, for example, the center has three adjacent $\mathrm{F}^{-}$vacancies with equilateral triangular symmetry in a (111) plane. Each vacancy binds one electron donated by neighboring $\mathrm{Na}^{+}$ions. Another alternate realization is a quantum well with triangular shape [8], gated to hold three electrons, or three identical closely-coupled quantum dots in triangular geometry, gated to hold one electron each.

Because we want our model to be relevant to $\mathrm{Nb}_{N}$ clusters, we must add some additional physics. We consider all distortions of the triangle in linear approximation (Sec. III). These have a particular importance because of a Jahn-Teller instability and a Longuet-HigginsBerry phase that inverts the order of singly and doubly degenerate levels (Sec. IV), and restores the ground-state degeneracy. We also consider rotations in space, but, for simplicity, only in two dimensions (Sec. V). Additional complications coming from higher order couplings, from rotations in the third dimension (these do not alter anything fundamental), and from antisymmetry of the nuclear wavefunction (the $\mathrm{Na}$ nucleus is a spin $3 / 2$ Fermion) are omitted for now. We plan to discuss some of these in a subsequent paper [9] which will focus on the $\mathrm{Na}_{3}$ trimer.

\section{DIPOLE MOMENT OF UNDISTORTED TRIMER}

The minimal toy model has three electrons which live in a Hilbert space of three $s$ orbitals $|n\rangle, n=0,1,2$ located on "atoms" which define vertices of a triangle. This crudely represents the outer electrons of an alkali or noble metal trimer $\mathrm{X}_{3}$. The subsequent paper will make contact with the specific case of $\mathrm{Na}_{3}$.

We define positions of X nuclei as $\vec{R}_{n}, n=0,1,2$. We write $\vec{R}_{n}=\hat{\Omega} \vec{r}_{n}$, where $\vec{r}_{n}=\vec{r}_{n 0}+\vec{u}_{n}$ give positions of nuclei in an internal reference frame of the molecule. The orientation of the molecular frame with respect to the laboratory frame is given by an $S O(3)$ matrix $\hat{\mathbf{\Omega}}$ which can be parameterized by three Euler angles. We choose the internal coordinates $x, y, z$ such that the molecule lies in the $x y$ plane. The atoms have small displacements $\vec{u}_{n}$ relative to equilateral triangular positions $\vec{r}_{n 0}=r_{0} \hat{\xi}_{n}$. The equilateral vertices are labeled by the unit vectors $\hat{\xi}_{n}$ where $\hat{\xi}_{0}$ lies in the molecular $\hat{x}$ direction, and 1,2 are rotated by $\pm 120^{\circ}$ around $\hat{z}$ as shown in Fig. 1]

The electrons are described by the tight-binding model

$$
H_{\mathrm{el}}=-\sum_{n=0}^{2}\left(t_{n, n+1} c_{n}^{\dagger} c_{n+1}+\text { c.c. }\right)+H_{\mathrm{int}}
$$

where $c_{n}$ is the annihilation operator of an electron on the $s$-orbital of the $n$-th atom. Coulomb interactions are mostly ignored. The effects of an on-site Hubbard interaction $H_{\text {int }}$ are considered in Appendix B The hopping

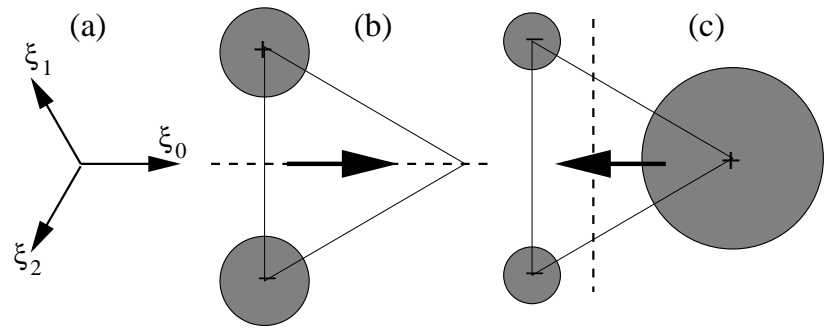

FIG. 1: (a) The unit vectors $\hat{\xi}_{n}$ which locate the undistorted positions in the molecular frame. (b) The conventional real basis function $\psi_{2}$ and (c) $\psi_{3}$ for the doubly degenerate $E^{\prime}$ orbitals which diagonalize $H_{\mathrm{el}}$ when the molecule is undistorted. Nodal planes are shown as dashed lines. If either of these states is occupied once and the other is empty, the atom will have a dipole moment $\pm \mu_{0}$, where $\mu_{0}=e r_{0} / 2$ and $r_{0}$ is the radial distance from the center to any atom. This dipole is shown as the bold vector.

matrix elements $t_{n, n+1}$ depend on the distance between atoms $\left|\vec{r}_{n}-\vec{r}_{n+1}\right|$.

The interaction with the external electric field $\vec{F}$ can be represented by

$$
H_{F}=-\vec{F} \cdot(\hat{\mathbf{\Omega}} \vec{\mu})=-\left(\hat{\mathbf{\Omega}}^{-1} \vec{F}\right) \cdot \vec{\mu}=-\vec{F}_{m} \cdot \vec{\mu},
$$

where the electric dipole moment in the molecule reference frame is given by

$$
\vec{\mu}=-\sum_{n=0}^{2} e \vec{r}_{n} c_{n}^{\dagger} c_{n}
$$

The factor $\vec{F}_{m}=\hat{\Omega}^{-1} \vec{F}$ is the electric field $\vec{F}$ as seen in the molecule reference frame.

For the rest of this section we take the molecule to be stationary, so that the lab frame coincides with the molecule frame $(\hat{\boldsymbol{\Omega}}=1)$, and neglect distortions $\left(\vec{u}_{n}=\right.$ $0)$. Therefore $t_{n, n+1}$ is a constant, $t_{0}$. For $\mathrm{Na}_{3}$, the hopping matrix element $t_{0}$ is about $0.4 \mathrm{eV}$ [10]. Eq. (1) is the $N=3$ case of the symmetric $N$-atom ring, treated in Hückel theory. The states follow from $N$-fold rotational symmetry

$$
|k\rangle=\frac{1}{\sqrt{N}} \sum_{n} e^{i 2 \pi k n / N}|n\rangle .
$$

The dimensionless wavenumber $k=-1,0,1$, defined modulo $N=3$, labels the energy levels. The energy eigenvalues are $\epsilon_{k}=-2 t_{0} \cos (2 \pi k / N)$. The lowest state $k=0$, of energy $-2 t_{0}$, is bonding (has no nodes), and is twice occupied. The other two states, $k= \pm 1$, are degenerate with energy $+t_{0}$ and hold one electron.

Now consider the electrical dipole operator (3). For a single electron in the orbital $|n\rangle$, the dipole operator has expectation value $\langle n|\vec{\mu}| n\rangle=-e \vec{r}_{n 0}$. The eigenstates $|k\rangle$ have equal charge on all atoms and therefore zero net dipole. However, there are off-diagonal matrix elements 
obeying a selection rule $\Delta k= \pm 1$,

$$
\langle k+1|\vec{\mu}| k\rangle=-\mu_{0}(\hat{x}-i \hat{y}),
$$

where $\mu_{0}=e r_{0} / 2$, and $\hat{x}, \hat{y}$ are unit vectors in the molecule reference frame. The matrix element $\langle k|\vec{\mu}| k+1\rangle$ is the complex conjugate of the one shown. A special property of the trimers with half-filled valence shells is that there are part-filled degenerate states with $k= \pm 1$. The selection rule $\Delta k= \pm 1$ does not exclude the matrix element $\langle k=-1|\vec{\mu}| k=1\rangle$ because -1 is the same as 2. Thus there is an off-diagonal dipole matrix element in the degenerate electronic ground state manifold. The $-\vec{\mu} \cdot \vec{F}_{m}$ coupling term, Eq. (2), is a $2 \times 2$ matrix in the subspace $|k=-1\rangle,|k=1\rangle$, namely

$$
\mathcal{H}_{F}^{\text {undist }}=-\mu_{0} \vec{\sigma} \cdot \vec{F}_{m}
$$

where $\vec{\sigma}=\sigma_{x} \hat{x}+\sigma_{y} \hat{y}$ and $\sigma_{x}$ and $\sigma_{y}$ are the usual offdiagonal Pauli matrices. The eigenvalues split linearly, $\Delta \epsilon= \pm \mu_{0}|\vec{F}|$, where it is important that the component $F_{m z}$ of $\vec{F}$ perpendicular to the plane of the molecule is omitted (that is, $|\vec{F}|$ is $\left(F_{m x}^{2}+F_{m y}^{2}\right)^{1 / 2}$.)

One might expect that other ring molecules with odd numbers of atoms, like $\mathrm{C}_{5} \mathrm{H}_{5}$, would also have this effect. Indeed, there is a dipole in the degenerate manifold $\mid k=$ $2\rangle,|k=-2\rangle$ for this case (since -2 is the same as 3 modulo $5)$, but this level is empty and there is no dipole in the partly filled $|k=1\rangle,|k=-1\rangle$ manifold. One might also be surprised that the Cartesian elements of the electrical dipole operator are represented by non-commuting $2 \times 2$ matrices, since these are commuting operators in a full theory. In the full three-dimensional space of the three $s$ states, the matrices $\mu_{x}, \mu_{y}, \mu_{z}$ commute, but in the $k=$ \pm 1 degenerate subspace relevant to low energy physics, they do not.

One can visualize this dipole moment by using the real linear combinations $\psi_{2}=(|k=1\rangle-|k=-1\rangle) / \sqrt{2} i$ and $\psi_{3}=(|k=1\rangle+|k=-1\rangle) / \sqrt{2}$, the conventional $E^{\prime}$ basis states with amplitudes $(0,1,-1) / \sqrt{2}$ and $(2,-1,-1) / \sqrt{6}$ on atoms $(0,1,2)$ shown on Fig. 1] These states have diagonal dipole expectation values $\pm \mu_{0} \hat{x}$ and off-diagonal elements $\mu_{0} \hat{y}$.

It is worth commenting on the magnetic properties of this model. The unpaired electron has a net spin. This couples weakly, through the spin-orbit interaction, to orbital angular momentum. The undistorted molecule has a large orbital magnetic moment. If the discrete three-membered ring were replaced by a 1 -d continuum (in the spirit of the free-electron approximation for bulk Na metal), the orbital moment would be $\vec{\mu}_{\text {orb }}=k \mu_{B} \hat{z}$ where $k= \pm 1$ is the quantum number of the outer unpaired electron. The free electron approximation gives the right symmetry and order of magnitude, but is not quantitatively correct. A correct calculation will give $\vec{\mu}_{\text {orb }}=k \gamma \mu_{B} \hat{z}$ where we estimate $\gamma \approx 0.3$ since the moment must be approximately $2 \pi e \omega A$ with $A$ the area of the molecule, and $2 \pi \omega$ is the classical frequency of electron rotation, approximately $\omega=t_{0} / \sqrt{3} \hbar$. In the next sections we find that distortions quench the orbital magnetic moment. For this reason we are not concerned with computing $\gamma$ which would have required additions to the model. Unlike the magnetic dipole moment, distortions reduce the electrical dipole moment only by a factor of 2. The "Bloch-sphere" gives a convenient representation of the magnetic and electric dipoles in this model, and is discussed in Appendix $\mathrm{A}$

\section{STATIC DISTORTIONS AND ADIABATIC LONGUET-HIGGINS PHASE}

To describe small distortions, consider the first order expansion in $\vec{u}_{n}=\vec{r}_{n}-r_{0} \hat{\xi}_{n}$. The hopping matrix is expanded to first order around the undistorted atom separation $\sqrt{3} r_{0}$,

$$
\begin{aligned}
t_{n, n+1} & =t_{0}-\frac{g}{\sqrt{3}}\left(\left|\vec{r}_{n}-\vec{r}_{n+1}\right|-\sqrt{3} r_{0}\right) \\
& =t_{0}-\frac{g}{3}\left(\vec{u}_{n+1}-\vec{u}_{n}\right) \cdot\left(\hat{\xi}_{n+1}-\hat{\xi}_{n}\right),
\end{aligned}
$$

where $g$ is the electron-phonon coupling.

To completely specify three atomic coordinates $\vec{R}_{n}$ requires nine numbers. Three give the position of the center of mass, and describe rigid translations of the molecule which separate out and do not contribute to Eq. (7). Three (e.g., Euler angles) locate the spatial orientation of the plane of the molecule, and the rotation angle of the molecule in the plane. These rotations, which also do not contribute to Eq. (7), will be discussed later. Finally, three parameters, chosen as amplitudes of three orthogonal normal modes of distortion, give the vibrations which do appear in Eq. (17).

The normal modes of an equilateral triangle comprise a symmetric ( $A_{1}^{\prime}$, or "breathing") mode and a doubly degenerate $\left(E^{\prime}\right)$ mode. Their amplitudes are conventionally [11, 12] called $\left(Q_{1}, Q_{2}, Q_{3}\right)$. Fig. 2] introduces a second trio of unit vectors $\hat{v}_{n}$. Like the previously introduced set $\hat{\xi}_{n}$, these vectors lie at $120^{\circ}$ to each other; they add to zero and are orthogonal to all translations and rotations and to the breathing mode eigenvectors $\hat{\xi}_{n}$.

The atomic coordinates omitting rotations and translations are

$$
\begin{aligned}
& \vec{r}_{n}=\left(r_{0}+Q_{1}\right) \hat{\xi}_{n}+Q \hat{v}_{n} \\
& \hat{\xi}_{n}=(\cos (2 \pi n / 3), \sin (2 \pi n / 3), 0)^{\dagger} \\
& \hat{v}_{n}=(\cos (\alpha-2 \pi n / 3), \sin (\alpha-2 \pi n / 3), 0)^{\dagger}
\end{aligned}
$$

where the dagger indicates the transpose of the row vector, which is a column vector. The amplitude $Q=$ $\sqrt{Q_{2}^{2}+Q_{3}^{2}}$ [13] and angle $\alpha=\tan ^{-1}\left(Q_{3} / Q_{2}\right)$, shown on Fig 2] parameterize the $E^{\prime}$ distortion. We will also assume that $Q_{1}, Q \ll r_{0}$ so that molecule is close to its symmetric "equilibrium" (see below) configuration $Q_{1}=Q=0$ or $\vec{r}_{n 0}=r_{0} \hat{\xi}_{n}$.

Vibrations are especially important because the orbitally degenerate ground state is Jahn-Teller unstable. 


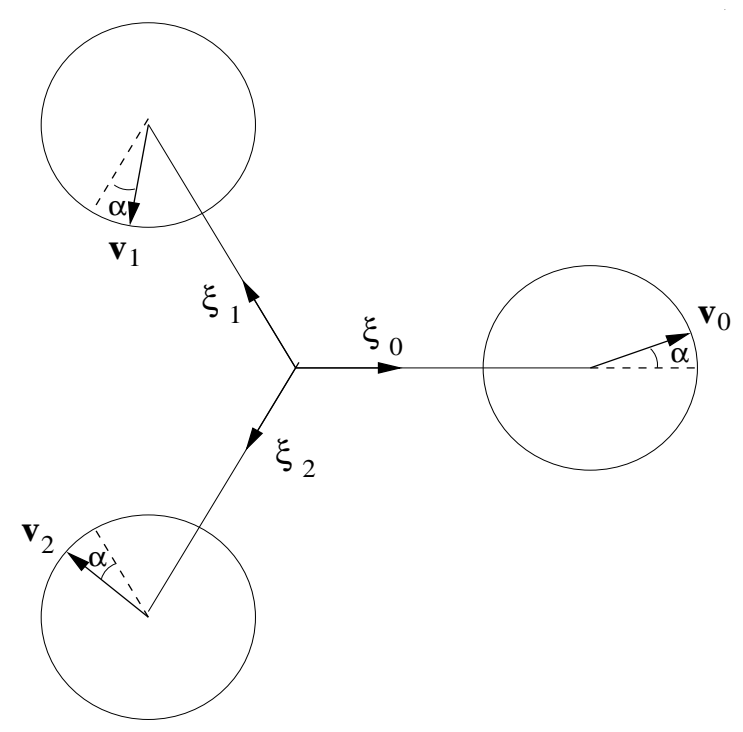

FIG. 2: Geometry of the distorted trimer. Atoms are at coordinates $\vec{r}_{n}=\left(r_{0}+Q_{1}\right) \hat{\xi}_{n}+Q \hat{v}_{n}$. When $Q=0$ the molecule has full $\mathrm{D}_{3 h}$ symmetry. When the angle $\alpha=0$, the vectors $\vec{v}_{n}$ lie along the dashed lines and describe a symmetric stretch which makes an isosceles distortion. This is the mode called $\mathrm{Q}_{2}$ by Van Vleck. When $\alpha=\pi / 2$, the vectors $\vec{v}_{n}$ give the asymmetric stretch $\mathrm{Q}_{3}$. These two patterns form the conventional basis for the doublet $E^{\prime}$ vibrations. Rather than $\left(Q_{2}, Q_{3}\right)$, we use the parameters $(Q, \alpha)$ to describe the $E^{\prime}$ vibrations. Note that the three vectors $\vec{v}_{n}$ lie at $120^{\circ}$ and add to zero, independent of $\alpha$. Note also that when $\alpha=120^{\circ}$, the distortion is isosceles with atom 2 at the vertex, and when $\alpha=240^{\circ}$, the isosceles distortion has atom 1 at the vertex.

The doublet $E^{\prime}$ distortions lift the degeneracy, lowering the energy of one of the doublet $E^{\prime}$ electron states. The latter is singly occupied in the ground state, thus lowering the energy of the molecule. In this section we make the static approximation. We assume that the trimer is distorted so that $Q_{1,2,3} \neq 0$ and solve electronic part of the problem in the presence of this static distortion. First, project Hamiltonian (11) onto the doubly degenerate ground state of an undistorted trimer. This projected Hamiltonian gives the electron-phonon term acting in the subspace of $(|k=-1\rangle,|k=+1\rangle)$ states as

$$
\mathcal{H}_{\mathrm{ep}}=g Q_{1} 1-g\left(Q_{2} \sigma_{x}-Q_{3} \sigma_{y}\right) .
$$

The "breathing" amplitude $Q_{1}$ shifts the degenerate levels uniformly. It also shifts the doubly occupied $|k=0\rangle$ level. Both these shifts will be absorbed into redefining the size $r_{0}$ to be the true radius of the molecule. The $\left(Q_{2}, Q_{3}\right)$ vibrations split the degenerate single-particle energies by $\pm g Q$ where $Q$ is the magnitude of the $\left(Q_{2}, Q_{3}\right)$ distortion. The splitting does not depend on the angle $\alpha$. The "lower Jahn-Teller" electron orbital (which is the lower eigenstate of Eq. 111, occupied with unit probability in adiabatic approximation) has the wave- function

$$
\begin{aligned}
\psi_{\mathrm{ad}} & =\frac{1}{\sqrt{2}}\left(e^{-i \alpha / 2}|k=1\rangle+e^{i \alpha / 2}|k=-1\rangle\right) \\
& =\sin (\alpha / 2) \psi_{2}+\cos (\alpha / 2) \psi_{3} .
\end{aligned}
$$

Here the first form of $\psi_{\text {ad }}$ uses the basis of Eq. (4) while the second form uses the basis of real functions shown in Fig. 1] For $\alpha=0$ the distortion is an acute isosceles triangle and the lower Jahn-Teller state is $\psi_{3}$ as shown in Fig. 11 panel (c). For $\alpha=180^{\circ}$, the distortion is obtuse, and the lower state is $\psi_{2}$. The node of the lower state cuts the longer bond, leaving the shorter bond nodeless.

The effective interaction with the external electric field can be obtained from (6]12) as

$$
\begin{aligned}
\mathcal{H}_{F} & =\left\langle\psi_{\text {ad }}\left|\mathcal{H}_{F}^{\text {rigid gs }}\right| \psi_{\text {ad }}\right\rangle \\
& =-\mu_{0} \vec{F}_{m} \cdot(-\vec{x} \cos \alpha+\vec{y} \sin \alpha) .
\end{aligned}
$$

The state $\psi_{\text {ad }}$ has an electrical dipole $\left\langle\psi_{\text {ad }}|\vec{\mu}| \psi_{\text {ad }}\right\rangle=\mu_{0} \hat{P}$, where the unit vector $\hat{P}$ is $\left(-Q_{2} \hat{x}+Q_{3} \hat{y}\right) / Q$. A correction of order $\left(Q / r_{0}\right) \cos (3 \alpha)$ to the dipole will also occur because the values of the vectors $\vec{r}_{n}$ in Eq. (3) change under distortion. However, effects ignored in our model make similar size corrections, so we ignore this. The dipole magnitude $\mu_{0}$ is the same as for the undistorted $\mathrm{D}_{3 h}$ case, but it points in a fixed direction. For $\alpha=0$, the distortion is acute and the ground state wavefunction the same as Fig. 1(c), with $\vec{\mu}$ in the $-\hat{x}$ direction. As $\alpha$ increases in a counter-clockwise sense, the negative charge of the last electron circulates clockwise (corresponding to current moving counterclockwise). The dipole $\vec{\mu}$ counterrotates as $\alpha$ rotates. We have lost the quantum nature of the dipole, which now orients classically. However, quantizing the distortional degrees of freedom will restore the quantum aspect. The orbital magnetic dipole is quenched in the adiabatic ground state, since the $k= \pm 1$ components are included in $\psi_{\text {ad }}$ with equal magnitude. Quantizing distortions will restore the magnetic dipole only very weakly.

Notice that the adiabatic wavefunction Eq. (12) changes sign when the molecule is taken once around the point $Q=0$ of "conical intersection" of the upper and lower Jahn-Teller energy surfaces. That is, when $\alpha$ increases by $2 \pi, \psi_{\text {ad }}$ changes sign. This adiabatic phase $\pi$ picked up by $\psi_{\text {ad }}$ is known as the LonguetHiggins phase 14] or as Berry's phase [15]. Important consequences of this for the quantized distortional states and for the electric dipole are worked out in the next section.

\section{QUANTIZED VIBRATIONS ALTERED BY LONGUET-HIGGINS PHASE}

The full Hamiltonian of the molecule can be written as

$$
\mathcal{H}=H_{\text {ions }}+H_{\text {pot }}+H_{\text {el }}+H_{F},
$$


where

$$
H_{\text {ions }}=-\frac{\hbar^{2}}{2 M} \sum_{n=0}^{2} \frac{\partial^{2}}{\partial \vec{R}_{n}^{2}},
$$

is the kinetic energy of the $\mathrm{Na}^{+}$ions. The potential energy is approximated by central springs,

$$
\begin{aligned}
H_{\mathrm{pot}} & =\sum_{n=0}^{2} \frac{K}{2}\left(\left|\vec{r}_{n}-\vec{r}_{n+1}\right|-\sqrt{3} r_{0}\right)^{2} \\
& \approx \frac{K}{2}\left[3 Q_{1}^{2}+\frac{9}{2} Q^{2}\right] .
\end{aligned}
$$

We use cyclic notations $n+3 \equiv n ; H_{e l}, H_{F}$ are Hamiltonians (12) of the valence electrons and of coupling to an external electric field $\vec{F}$.

In adiabatic approximation one looks for the total wave function of electrons and ions in the form of a product $\Psi\left(\vec{R}_{n}\right) \psi_{\text {ad }}$. Then the effective Hamiltonian acting on $\Psi\left(\vec{R}_{n}\right)$ is obtained from Eqs. (1115116). It acquires an induced "potential energy" $\left\langle\psi_{\text {ad }}\left|\mathcal{H}_{\text {ep }}\right| \psi_{\text {ad }}\right\rangle=g Q_{1}-g Q$ and can be written in the reference frame of the molecule as the sum of the kinetic and potential energy of vibrational modes,

$$
\begin{aligned}
\mathcal{H}_{\mathrm{ad}} & =\mathcal{H}_{A}+\mathcal{H}_{E^{\prime}} \\
\mathcal{H}_{A} & =\frac{3 M}{2} \dot{Q}_{1}^{2}+\frac{3 K}{2} Q_{1}^{2}+g Q_{1} \\
& \rightarrow \frac{3 M}{2} \dot{Q}_{1}^{2}+\frac{3 K}{2} Q_{1}^{2}-\frac{g^{2}}{6 K} \\
\mathcal{H}_{E^{\prime}} & =\frac{3 M}{2}\left(\dot{Q}_{2}^{2}+\dot{Q}_{3}^{2}\right)+\frac{9 K}{4} Q^{2}-g Q,
\end{aligned}
$$

where $M$ is the mass of the $\mathrm{Na}$ atom. The second form of $\mathcal{H}_{A}$, Eq. (17), results from the redefinition $r_{0}-g / 3 K \rightarrow r_{0}$ of the size of the trimer, and the corresponding redefinition $Q_{1}+g / 3 K \rightarrow Q_{1}$. The breathing mode frequency $\omega_{A}$ in this model is $\sqrt{K / M}$; in $\mathrm{Na}_{3}$, multireference configuration interaction calculations give this to be about $17 \mathrm{meV}$ [16], while experiment 17] suggests $16 \mathrm{meV}$, which implies $K \approx 1.5 \mathrm{eV} / \AA^{2}$.

Unlike Eq. (17), Eq. (18) cannot be simplified by a shift of origin. Rather than being a simple shifted parabola, the two-dimensional potential surface of Eq. (18) has a cusp at the origin. This is the "conical intersection" between the lower energy adiabatic energy surface and the Jahn-Teller-split higher energy surface, which replaces $-g Q$ by $+g Q$ in Eq. (18). The higher surface does not enter in adiabatic approximation. The potential energy of Eq. (18) is cylindrically symmetric around $Q_{2}=Q_{3}=Q=0$, and has the form of a "Mexican hat." The value $Q_{\min }=2 g / 9 \mathrm{~K}$ minimizes this potential in the radial direction in $\left(Q_{2}, Q_{3}\right)$-space, and the energy is lowered by the Jahn-Teller energy $-g^{2} / 9 K$. The frequency of radial oscillation is $\omega_{Q}=\sqrt{3 K / 2 M}$, but for angular motion there is no restoring force, $\omega_{\alpha}=0$. The free motion of the variable $\alpha$ is called "pseudorotation." When the coordinate $\alpha$ increases monotonically, the molecular coordinates $r_{n}$ of the atoms move in counter-clockwise paths in the molecular $x, y$ plane (see Fig. 2) accompanied by no collective rotation, but by true angular momentum, which of course must be quantized.

Quantum chemical calculations for $\mathrm{Na}_{3}$ give a somewhat different picture, because the distortion $(\sim 10 \%)$ is not very small, and as a result there is significant warping of the path of least energy on the energy surface in the $Q_{2}, Q_{3}$ plane surrounding the conical intersection at the origin. Most calculations find the minimum to be an obtuse isosceles triangle $\left(\alpha=180^{\circ}\right.$ or equivalently $\alpha= \pm 60^{\circ}$ on Fig. 22). Saddle points, representing barriers for pseudorotation, occur at the acute positions $\left(\alpha=0^{\circ}\right.$ or equivalently $\left.\pm 120^{\circ}\right)[18]$. The calculation of von Busch et al. [16] gives the average distortion $Q_{\text {min }}=2 g / 9 K \approx 0.25 \AA$. which implies that the coupling constant $g$ is about $1.7 \mathrm{eV} / \AA$. The same calculation gives the energy minimum to be $97 \mathrm{meV}$ and the barrier height to be $25 \mathrm{meV}$, higher than the breathing phonon energy, and sufficient to strongly inhibit pseudorotation. In our model the energy minimum $-g^{2} / 9 \mathrm{~K}$ is about $200 \mathrm{meV}$, twice the quantum-chemical value, and there is no barrier in leading order.

The kinetic part of Eq.(18) can be written as $(3 M / 2) \dot{Q}^{2}+\left(I_{Q} / 2\right) \dot{\alpha}^{2}$, in separated radial and pseudorotational parts, with pseudorotational moment of inertia $I_{Q}=3 M Q^{2}$. The zero-point amplitude of radial oscillation $\left(\hbar^{2} / 6 K M\right)^{1 / 4}$ is small enough compared with $Q_{\text {min }}$ that we can approximate the pseudorotational moment of inertia by the constant $I_{Q}=3 M Q_{\min }^{2}$. Then the pseudorotational wavefunction (in our approximation with no restoring force) is $\psi_{\text {ps-rot }}=\exp (i m \alpha)$ and the energy is $\hbar^{2} m^{2} / 2 I_{O}$. As pointed out long ago by Longuet-Higgins et al. [19], the pseudorotational quantum number $m$ must take half-integer values. This is because the total wavefunction $\psi_{\text {ps-rot }}(Q, \alpha) \psi_{\text {ad }}$ must be a single-valued function of the coordinate $\alpha$. We already noticed that the adiabatic electron wave-function Eq. (12) changes sign under one pseudorotational cycle. Therefore the function $\psi_{\text {ps-rot }}$ must also change sign when $\alpha$ is increased by $2 \pi$. The ground state, with pseudorotational quantum $m= \pm 1 / 2$, is thus still doubly degenerate. The electronic orbital degeneracy was lifted by the Jahn-Teller distortion $Q \rightarrow Q_{\min }$, but left and right pseudorotating states restore the degeneracy of the combined electronic and atomic system. This restoration of degeneracy is the discrete system analog of restoration of translation invariance by phase solitons in one-dimensional molecular chains [20].

The wavefunctions $\psi_{m}$ of this adiabatic vibrational ground state are products of the pseudorotational part $\exp (i m \alpha)$ times the electronic part $\psi_{\text {ad }}$ (Eq.(12), also multiplied by the ground state harmonic oscillator functions for breathing $Q_{1}$ and radial oscillation $Q$. The dipole operator in this $2 \mathrm{~d}$ manifold is obtained by pro- 
jecting Eq. (13) onto $|m= \pm 1 / 2\rangle$

$$
\mathcal{H}_{F}^{\text {ad vib gs }}=-\frac{\mu_{0}}{2} \vec{\tau} \cdot \vec{F}_{m},
$$

where $\vec{\tau}=\tau_{x} \hat{x}+\tau_{y} \hat{y}$ are Pauli matrices acting in the space of pseudorotational states $|m=1 / 2\rangle$ and $\mid m=$ $-1 / 2\rangle$. The eigenstates $\pm 1 / 2$ of pseudo-angular momentum $\left(-i \partial_{\alpha}\right)$ have left and right circulating dipolar charge currents. Unlike the undistorted molecule, the frequency of circulation is no longer $t_{0} / \hbar$, but is now $\hbar / I_{Q}$, a much smaller number. Therefore the orbital magnetic dipole moment is restored, except with a much smaller value. There is no diagonal expectation value of the electric dipole, only an off-diagonal value, just as in the undistorted $\mathrm{D}_{3 h}$ case, but reduced by 2 , from $\mu_{0}$ to $\mu_{0} / 2$. Thus the half-filled outer orbital of the symmetric triangular molecule has a quantum dipole which persists under quantization of molecular distortions. A field $\vec{F}_{m}$ applied in the plane of the molecule splits the levels linearly, $\pm \mu_{0}\left|\vec{F}_{m}\right| / 2$. The eigenstates in the field are linear combinations of the left and right pseudorotators whose moment is distributed in the plane of the molecule with probability distribution $\cos ^{2}\left(\alpha_{F}\right)$ where $\alpha_{F}$ is the angle measured from the projection of the field onto the plane of the molecule. This cosine distribution is what causes the reduction of $\mu_{0}$ by 2 .

In principle this is not fundamentally altered by a warping potential like $\left(V_{0} / 2\right)(1+\cos (3 \alpha))$, because tunneling along the pseudorotational minimum energy path preserves 3 -fold symmetry and guarantees a degenerate ground state 21. Pseudorotational quantum numbers $m$ are still half-integer quantized, but defined modulo 3 , so that for example $m=3 / 2$ and $m=-3 / 2$ are the same and this state is singly degenerate, while $m= \pm 5 / 2$ is a doublet belonging to the $m=\mp 1 / 2$ representation. The splitting $\hbar \omega_{\text {pseudorot }}$ between the $m= \pm 1 / 2$ ground state and the $m=3 / 2$ excited state gets reduced in frequency from $\omega=\hbar / I_{Q}$ by a Franck-Condon-type overlap factor governing the tunneling probability. Thus, warping can greatly reduce the orbital magnetic dipole, which is proportional to the pseudorotational splitting. Also, thermal mixing of pseudorotational states will smear any effect for $k_{B} T>\hbar \omega_{\text {pseudorot }}$.

\section{QUANTIZED ROTATIONS}

Now we must deal with real rotations of the molecule in space, which adds a lot of complexity, as it does even for a fixed dipole. A full 3-d analysis does not fundamentally change things from a much simpler analysis of rotations in 2-d, so we confine ourselves to 2-d rotations in this paper. Here we describe how 2-d rotations alter both a fixed dipole and our quantum pseudorotator. We work in the limit where the molecule stays in its vibrational ground state, but has pseudorotational excitations.

The Hamiltonian for our pseudorotating quantum dipole $(\mathrm{PQD})$ is

$$
\begin{aligned}
\mathcal{H}_{2 \mathrm{~d} \mathrm{PQD}} & =\frac{\hbar^{2}\left(\pi_{\alpha}+\pi_{\psi}\right)^{2}}{2 I_{0}}+\frac{\hbar^{2} \pi_{\alpha}^{2}}{2 I_{Q}}+\frac{V_{0}}{2}(1+\cos 3 \alpha) \\
& -\mu_{0} F \cos (\psi+\alpha)
\end{aligned}
$$

Here $\psi$ is the Euler angle defining the orientation of the reference frame of the molecule with respect to lab frame. $\pi_{\alpha}$ and $\pi_{\psi}$ are dimensionless angular momenta which in quantum theory are $-i \partial_{\alpha}$ and $-i \partial_{\psi}$ and take half-integer and integer values correspondingly. The first term of (20) is the kinetic energy of rotation of the trimer as a whole with $I_{0}=3 M r_{0}^{2}$. The second term is the kinetic energy of pseudorotation, and the third term is the simplest model for the warped potential energy of pseudorotation, with minima at the obtuse isosceles positions, and barriers of height $V_{0}$ at the acute positions. The simplest version of our model has $V_{0}=0$. The last term is the coupling to the external electric field. It can be obtained from (13) by substituting $\vec{F}_{m}=F(\cos \psi,-\sin \psi)$.

Eq. (20), with $V_{0}$ set to zero, models a quantum electric dipole moment, free to rotate in $2 \mathrm{~d}$ space. We now calculate the observable dipole moment for a statistical ensemble at temperature $T$. This is $\langle\mu\rangle=-\partial \mathcal{F} / \partial F$, where $\mathcal{F}$ is the free energy of the dipole calculated at temperature $T$ with Hamiltonian (20). The temperature scales set by rotations and pseudo-rotations are $T_{\text {rot }}=\hbar^{2} / 2 I_{0} \ll T_{\mathrm{ps}-\text { rot }}=\hbar^{2} / 2 I_{Q}$. For $\mathrm{Na}_{3}$ these are approximately $0.1 \mathrm{~K}$ and $5 \mathrm{~K}$. We assume $T \gg T_{\text {rot }}$, which allows us to treat spatial rotations classically, but $T$ can be either above or below $T_{\mathrm{ps}-\mathrm{rot}}$.

Before proceeding to the quantum dipole (20) we first summarize the well known results for the classical dipole moment in an external electric field.

\section{A. Classical dipole}

The Hamiltonian of a $2 \mathrm{~d}$ fixed dipole is

$$
\mathcal{H}_{2 \mathrm{~d} \text { FD }}=\frac{\hbar^{2} \pi_{\psi}^{2}}{2 I_{0}}-\mu_{0} F \cos \psi
$$

where $I_{0}$ is the molecule's moment of inertia. This is the pendulum problem. The low-lying quantum eigenenergies of this system, calculated numerically, are shown in Fig. 3 .

The classical treatment is standard. After evaluating the partition function $Z=\exp (-\mathcal{F} / T)$ (we use units $k_{B}=1$ ), the classical average dipole is found by differentiating $\mathcal{F}$ to be

$$
\langle\mu\rangle=\mu_{0} \frac{\int_{-\pi}^{\pi} d \psi \cos \psi e^{\left(\mu_{0} F / T\right) \cos \psi}}{\int_{-\pi}^{\pi} d \psi e^{\left(\mu_{0} F / T\right) \cos \psi}}
$$

which saturates to $\mu_{0}$ at high field $F$ and equals $\chi F$ at low field, where $\chi=\mu_{0}^{2} / 2 T$ is the 2 -d version of the Debye-Langevin polarizability [3]. 


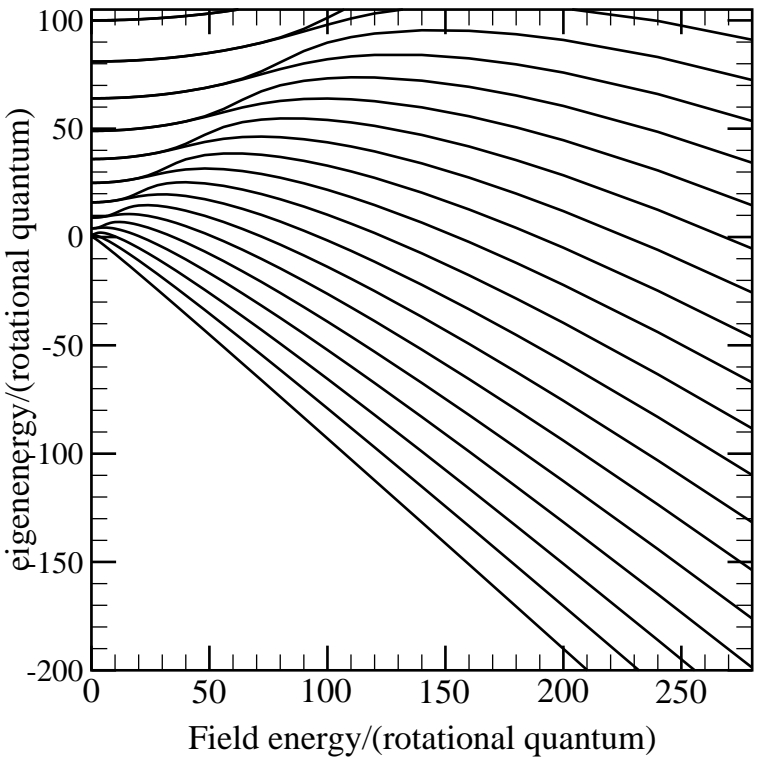

FIG. 3: Spectrum of low-lying energy eigenvalues of the 2$\mathrm{d}$ fixed dipole rotator. The field energy is $\mu_{0} F$ and the rotational quantum of energy is $T_{\text {rot }}=\hbar^{2} / 2 I$. The zero field axis has dimensionless eigenvalues equal to $m^{2}$ for $m=$ $0, \pm 1, \pm 2, \ldots$. Each level except $m=0$ is doubly degenerate, and the degeneracy is split by the field.

The results of the quantum treatment of this problem do not have a simple form. At high field $\left(\mu_{0} F \gg T\right)$ the dipole makes pendulum oscillations around the direction of the field. This is seen in the lower right part of Fig. 3 where there are evenly spaced levels at $\Delta E=\hbar \omega$ where the pendulum frequency $\omega=\sqrt{\mu_{0} F / I_{0}}$ increases as the field increases. At low field and low temperature, the quantum ground state is rotationally symmetric with a dipole induced by second-order coupling to the first rotationally excited states. In this limit the polarizability becomes $\mu_{0}^{2} /\left(\hbar^{2} / 2 I\right)$. If the field increases to values large compared with $T / \mu_{0}$, the eigenstates are mixtures of rotational levels. These mixtures give a full dipole moment, saturated at the value $\langle\mu\rangle=\mu_{0}$. We plot the dependence of dipole moment versus an applied electric field in Fig. 4. In different limits we have

$$
\begin{aligned}
\frac{\langle\mu\rangle_{2 \mathrm{~d} C D}}{\mu_{0}} & \approx \frac{\mu_{0} F}{T_{\text {rot }}} \quad\left(\mu_{0} F \ll T_{\text {rot }}\right) \\
& \approx \frac{\mu_{0} F}{2 T} \quad\left(T_{\text {rot }} \ll \mu_{0} F \ll T\right) \\
& \approx 1-\frac{T}{\mu_{0} F} \quad\left(T \ll \mu_{0} F \ll \frac{T^{2}}{T_{\text {rot }}}\right) \\
& \approx 1-\frac{1}{4} \sqrt{\frac{2 T_{\text {rot }}}{\mu_{0} F}} \quad\left(\mu_{0} F \gg \frac{T^{2}}{T_{\text {rot }}}\right),
\end{aligned}
$$

where the subscript "CD" means classical dipole (the rotations are treated quantum mechanically). The water molecule is a good example. Normally we think of a classical fixed dipole, but the quantum eigenstates in zero

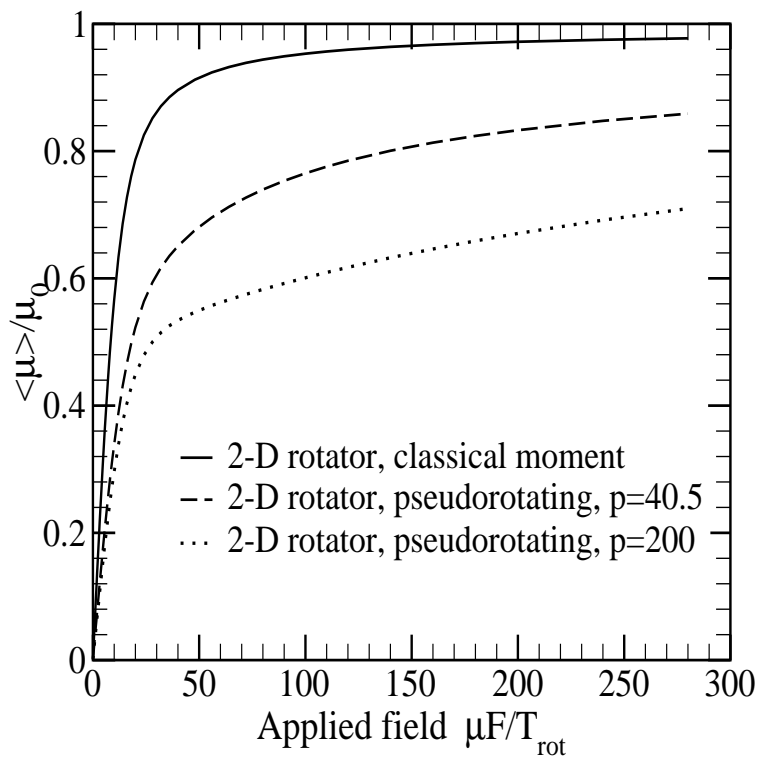

FIG. 4: Dipole moment of classical and quantum dipoles as a function of applied electric field, calculated at temperature $T=7.0 T_{\text {rot }}$. The quantum rotator has a dimensionless parameter $p=T_{Q} / T_{\text {rot }}$ which is set to 40.5 (dashed curve, reasonable for $\mathrm{Na}_{3}$ ) and to 200 (dotted curve).

field are rotating dipoles with no fixed average moment. In a typical chemical or biological environment, the environmental field $F$ is large, free rotations are hindered, and the dipole is exposed.

\section{B. Quantum dipole with free pseudorotations}

To emphasize the difference between a quantum dipole (20) and a fixed dipole (21), we choose the limit $V_{0}=0$, i.e., a quantum dipole with free pseudorotations. Eq.(20) with $V_{0}=0$ is a funny degenerate sort of double pendulum with two angular degrees of freedom. The quantum states at zero field $\vec{F}=0$ have eigenvalues

$$
\epsilon(\ell, m)=\hbar^{2}(\ell+m)^{2} / 2 I_{0}+\hbar^{2} m^{2} / 2 I_{Q}
$$

where the angular momentum quantum number $\ell$ takes integer values $0, \pm 1, \ldots$ and the pseudomomentum quantum number $m$ takes half-integer values $\pm 1 / 2, \pm 3 / 2, \ldots$. Because $I_{Q} \ll I_{0}$, the low-lying states have $m= \pm 1 / 2$. All eigenstates are 4-fold degenerate, even the ground state, because for either choice of $|m|$ there are two ways to get any allowed value of $|\ell+m|$. When the field is applied, each quadruplet level splits into two doublets. There are no allowed matrix elements of the field in any degenerate manifold of 4 states, so the initial splitting is quadratic in field, just as for water. If we assume that the temperature is low enough that only the lowest pseudorotational levels $m= \pm 1 / 2$ are excited, it is a good approximation to ignore the term in Eq.(20) involving $I_{Q}$. Then the 2-d Hamiltonian (20) separates into 


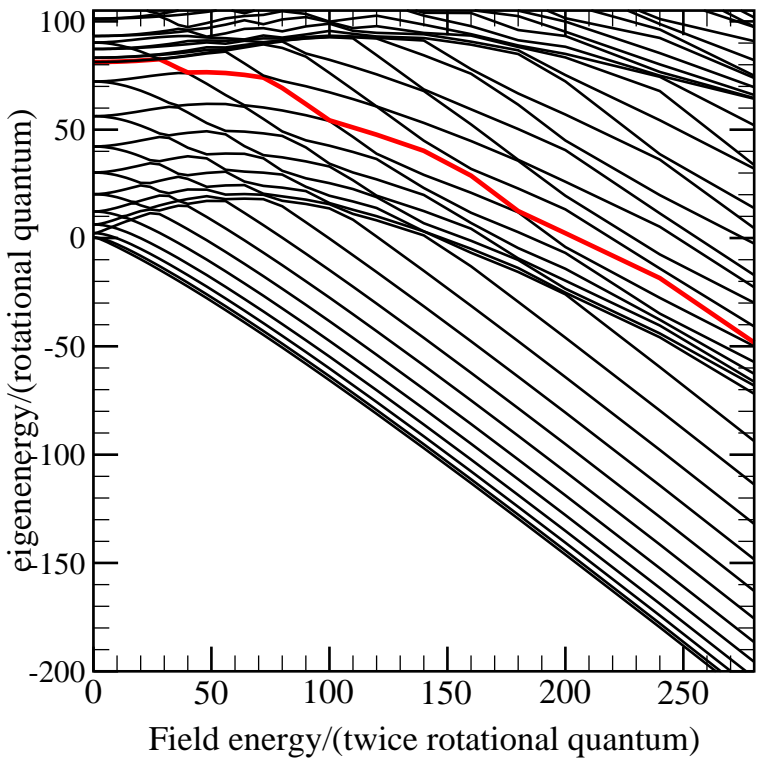

FIG. 5: Spectrum of low-lying energy eigenvalues of the 2$\mathrm{d}$ quantum dipole rotator. The field energy is $\mu_{0} F$ and the rotational quantum of energy is $\hbar^{2} / 6 M r_{0}^{2}$. The zero field axis has dimensionless eigenvalues equal to $(\ell+m)^{2}+$ $\left(r_{0} / q_{\min }\right)^{2} m^{2}$, with the zero-point pseudorotational quantum $\left(r_{0} / q_{\text {min }}\right)^{2}(1 / 2)^{2}$ subtracted out. The level in red at zero $F$ is the first with pseudorotational quantum number $|m|>1 / 2$. Each level at zero $F$ is quadruply degenerate, and the degeneracy is reduced to double by the field.

$2 \times 2$ matrices acting on subspaces formed by $\mid l, m=$ $1 / 2\rangle,|l-1, m=-1 / 2\rangle$. The ground state energy is $T_{\text {rot }}\left[5 / 4-\sqrt{1+\left(\mu_{0} F /\left(2 T_{\text {rot }}\right)\right)^{2}}\right]$, which implies a ground state dipole $\langle\mu\rangle=\left(\mu_{0} / 2\right)\left[\left(\mu_{0} F / 2\right) / \sqrt{\left(\mu_{0} F / 2\right)^{2}+T_{\text {rot }}^{2}}\right]$. For high fields and $T$ not too low $\left(\mu_{0} F \gg T \gg T_{\text {rot }}\right)$ the dipole is

$$
\langle\mu\rangle_{2 \mathrm{~d} \text { QD }} \approx \frac{\mu_{0}}{2}\left[1-\frac{2 T T_{\text {rot }}}{\left(\mu_{0} F / 2\right)^{2}}\right]
$$

Notice that the effect of temperature (when it is less than the field energy $\mu_{0} F$ ) is smaller on the quantum (pseudo- + real-) rotating dipole Eq.(25) than on the fixed rotating dipole Eq.(23); there is an extra small factor $T_{\text {rot }} /\left(\mu_{0} F / 2\right)$ which postpones thermal degradation of the dipole by rotation. The reason is evident in the difference between the spectra seen in Fig. 3 and Fig. 5. At higher fields $F$ and not too high excitation energy, the fixed dipole has energy levels that fan out to larger separations in higher fields, meaning lower slopes and lower dipoles in higher excited states, while the quantum dipole has energy levels whose slopes do not depend on excitation level. The fixed dipole levels correspond to pendulum oscillations of the dipole away from the field direction, whereas the quantum dipole levels correspond to free rotations (the energy levels are $\left.\hbar^{2}(\ell+1 / 2)^{2} / 2 I_{0}\right)$ of a molecule whose dipole is fixed in the direction of the field. The way this happens is that the dipole is not fixed in the axes of the molecule, but free to pseudorotate around these axes. Real rotations and pseudorotations give compensating changes in the dipole moment, leaving the moment fixed in space. This happens equally well for left and right rotations, so all levels are still doublets. The comparison between dependences of $\langle\mu\rangle$ vs. $F$ is presented in Fig. 目

\section{DISCUSSION AND CONCLUSIONS}

The main point of the present paper is the possible relevance to the issue of unusual polarity in metal clusters 1, 2]. Existing experiments on $\mathrm{X}_{3}$ molecules have not seen the effects described here. There are multiple possible reasons, including (a) not looking for quite the right effect, (b) need for lower temperature, (c) nuclear exchange symmetry, and (d) suppression by large barriers to pseudorotation. A subsequent paper will address some of these issues in the context of $\mathrm{Na}_{3}$. Here we speculate about the more general issues.

Suppose we discard triangular symmetry altogether, as in a mixed alkali trimer like $\mathrm{Li}_{2} \mathrm{Na}$ 22, or LiNaK. In the symmetric case, symmetry forced the conical intersection which preserved (by pseudorotation) the degenerate ground state and its quantum dipole. However, as explained by Wigner 14, 23], points of degeneracy, or conical intersections, are generically present [24] if there are enough free variables. In the last occupied molecular orbital level of LiNaK, the splitting of the two sublevels can be tuned to zero by adjustment of two parameters, since the Hamiltonian matrix can be written in this manifold as real symmetric $2 \times 2$. There are three such parameters, the normal mode amplitudes $\left(Q_{1}, Q_{2}, Q_{3}\right)$. Thus it is permitted to have a line in configuration space where the upper and lower energy surfaces touch and away from which they separate linearly. Consider the minimum energy point on this line, and also the global minimum of the lower energy surface. Away from the global minimum, the energy increases quadratically with three principal axes defining nearby ellipsoidal energy surfaces. Now begin moving along the axis of least energy increase, and continue following the bottom of this valley. It is possible that this valley will return to the global equilibrium after circling the line of accidental degeneracy. It is also possible that the path in this minimum valley lies always lower than the minimum energy found on the line of degeneracy. In this case, there is again a pseudorotation with an electronic-wavefunction sign change. The lowest energy pseudorotational state must therefore have a compensating phase change of $\pi$ when orbiting the degenerate line. Time-reversal symmetry then requires that an inequivalent reverse pseudorotation with opposite phase is degenerate. These two states should each drag a dipole moment in opposite directions once around the degeneracy line as the system moves adiabatically around the circuit. Thus a quantum dipole is generically permitted. Larger clusters of metal atoms have more complex en- 
ergy surfaces, and both features (1) conical intersections and (2) lack of inversion symmetry are generically likely. Therefore unusual dipoles whose properties are not fully classical may be expected. Possibly this is what Moro et al. [1, 2] observed.

Moro et al. observed a correlation between unusual dipoles and metals known in bulk form to have superconductivity caused by large electron-phonon coupling. Large electron-phonon coupling derives from a high electronic density of states and a rapid change of singleparticle energy eigenvalues with deformation. These are conditions favorable to the occurence of the dipole we have described. A high density of states means plentiful levels whose energies may accidentally cross at conical intersections. Sensitivity of eigenvalues to distortion means that the point of the cone of intersection can lie higher in energy than the surrounding landscape. Thus our picture contains the right ingredients. Further experiment and more accurate microscopic modelling will be needed to confirm it.

To conclude, we studied the formation of an electrical dipole moment in triangular molecules. Although, the classical dipole moment is forbidden for symmetric trimers it is formed due to the quantum degeneracy of the ground states. This dipole moment survives JahnTeller distortion of the molecule and keeps its "quantum nature" for small distortions. It is interesting to note that the quantum dipole moment makes a two-level system which can be manipulated by an external electric field. If it were possible to have a controlled interaction between such trimers, one could investigate the use of this two-level system for quantum computing purposes similarly to what was done for quantum qubits using persistent currents [25].

\section{ACKNOWLEDGEMENTS}

We thank W. de Heer for discussions of ref. 1. We acknowledge helpful suggestions from T. Baruah, W. Ernst, K. Lehmann, J. Muckerman, R. Porter, G. Scoles, and A. M. Stoneham. The work of PBA was supported in part by NSF grant no. DMR-0089492, and in part by a J. S. Guggenheim Foundation fellowship. Work at Columbia was supported in part by the MRSEC Program of the NSF under award no. DMR-0213574. Work of AGA was supported by the Alfred P. Sloan foundation, NSF grant DMR-0348358, and the Theory Institute of Strongly Correlated and Complex Systems at Brookhaven.

\section{APPENDIX A: BLOCH SPHERE}

It is convenient to use the Bloch sphere parameterization of the degenerate ground state $|g\rangle=(\alpha, \beta)^{t}=\alpha \mid k=$ $-1\rangle+\beta|k=1\rangle$. Define the unit vector $\vec{n}=\langle g|\vec{\sigma}| g\rangle$. The manifold of degenerate states of the two-level system (undistorted trimer) is represented then by two-

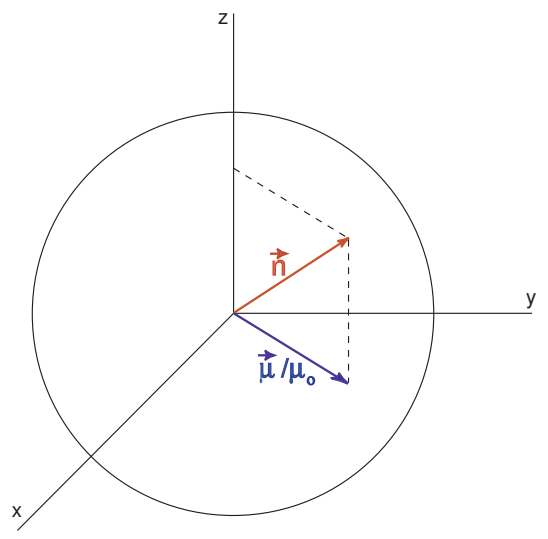

FIG. 6: In the Bloch sphere parameterization the ground states of two-level system correspond to the points of a unit sphere i.e., they can be labeled by a unit vector $\vec{n}$. For the undistorted trimer the $x y$ projection of this unit vector is proportional to the electric dipole moment while its $z$ component is proportional to the magnetic moment of the ground state.

dimensional sphere swept by the unit vector $\vec{n}$. The electric dipole moment $\vec{\mu}$ in the corresponding ground state is proportional to the projection $\vec{n}_{\perp}$ of $\vec{n}$ on the $x y$ plane $\vec{\mu}=\mu_{0} \vec{n}_{\perp}$.

The partly-occupied states $|k= \pm 1\rangle$ of the static trimer have an orbital magnetic moment perpendicular to the plane of the molecule which couples to a magnetic field $\vec{B}$. In the degenerate $E^{\prime}$ subspace, the coupling is

$$
\mathcal{H}_{B}=-\mu_{\mathrm{orb}} \sigma_{z} B_{z}
$$

where the moment $\mu_{\text {orb }}$ is $2 \pi e \omega A$ as previously discussed. In the Bloch sphere parameterization, the magnetic dipole moment is proportional to the projection of $\vec{n}$ onto the $z$ axis, and is equal to $\mu_{\mathrm{orb}} n_{z}$.

The value of the moment $\mu_{\text {orb }}$ for $\mathrm{Na}_{3}$ is about $0.3 \mathrm{Bohr}$ magnetons, if we ignore the barrier to free pseudorotation. The electrical moment $e r_{0} / 2$ is $0.98 \mathrm{e} \AA$ or $4.7 \mathrm{D}(1$ Debye unit $=0.208$ eA.) in our naive model. An unpublished density functional calculation [26] gives 2.3D. It is not surprising that the actual result is reduced, because the true molecular orbitals surely have a significant $p \sigma$ component missing from our model. Also, the on-site Coulomb repulsion causes a suppression of the dipole as is shown in the next appendix.

\section{APPENDIX B: EFFECT OF A HUBBARD TERM}

The Coulomb interaction between electrons is approximated by a Hubbard term of strength $U$,

$$
H_{i n t}=U \sum_{n=0,1,2}\left(c_{n \uparrow}^{\dagger} c_{n \uparrow}+c_{n \downarrow}^{\dagger} c_{n \downarrow}\right)^{2}
$$

The Hilbert space has dimension 20. There are 8 basis states with no vacant sites and 12 with one vacant site. 


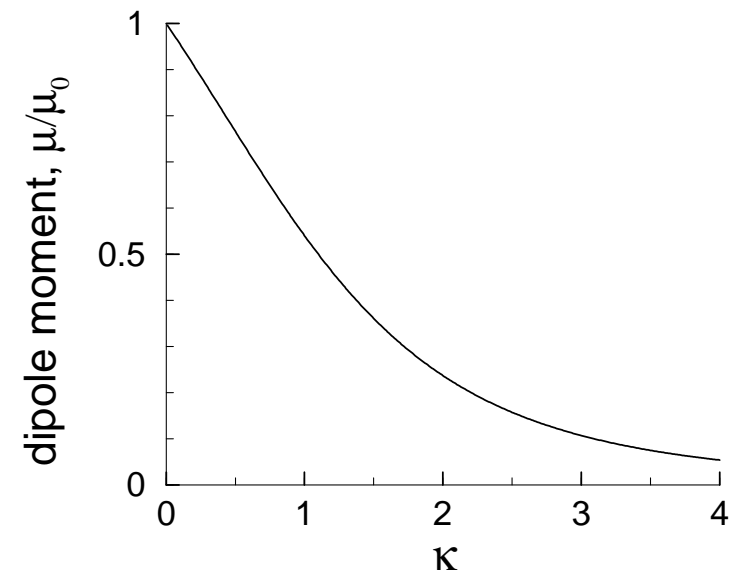

FIG. 7: The quantum dipole in units $e r_{0} / 2$ as a function of the dimensionless Hubbard strength $\kappa \equiv U / t_{0}$. The intercept coincides with the non-interacting Hückel model, while for $\kappa \gg 1$ it falls as $\kappa^{-3}$.

Total spin, spin $\hat{z}$-projection, and orbital (electronic) angular momentum are conserved. In the eight-dimensional $S=1 / 2$ and $S_{z}=1 / 2$ subspace, there are three states with angular momentum $k=1$, three with $k=-1$, and two with $k=0$ that have higher energy. Parity symmetry is not broken by the Hubbard term. The ground state is a $k= \pm 1$ doublet, as it was for the non-interacting model. Figure 7 shows the dependence of the quantum dipole on the dimensionless Hubbard parameter $\kappa \equiv U / t_{0}$. A factor of two suppression occurs when $U$ is of the same order as $t_{0}$. For $\kappa \ll 1$, the doubly degenerate ground state energy is $E_{0} \approx-3 t_{0}+(13 / 3) U$. The dipole operator has an off-diagonal matrix element $\langle-1|\vec{\mu}|+1\rangle$ between $k=-1$ and $k=+1$ states equal to $-\mu(\hat{x}-i \hat{y})$ with

$$
\mu \approx \mu_{0}\left[1-\frac{4}{9} \kappa\right], \quad \text { for } \kappa \ll 1
$$

This agrees with the non-interacting result, Eq. (5). For $\kappa \gg 1$, the doubly degenerate ground state has energy $E_{0} \approx 3 U-3 t_{0}^{2} / U$. The magnitude $\mu$ of the off-diagonal matrix element falls rapidly as shown in Fig. 7 going asymptotically as

$$
\mu \approx \frac{9}{2} \kappa^{-3} \mu_{0}, \text { for } \kappa \gg 1
$$

[1] R. Moro, X. Xu, S. Yin, and W. A. de Heer, Science 300, 1265 (2003).

[2] R. Moro, S. Yin, X. Xu, and W. A. de Heer, Phys. Rev. Letters 93, 086803 (2004).

[3] J. H. Van Vleck, Theory of Electric and Magnetic Susceptibilities (Oxford Univ. Press, Oxford, 1932) p. 184 and 307.

[4] C. H. Townes and A. L. Schawlow, Microwave Spectroscopy, Dover, New York, 1975, Ch. 10.

[5] ibid p.52.

[6] W. Klemperer, K. K. Lehmann, J. K. G. Watson, and S. C. Wofsy, J. Phys. Chem 97, 2413 (1993).

[7] A. M. Stoneham, Theory of Defects in Solids, Oxford Univ. Press, Oxford, 1975; Ch. 17.

[8] G. Zarand, A. Brataas, and D. Goldhaber-Gordon, Sol. State Commun. 126, 463 (2003).

[9] [Requist, Baruah, Abanov, Allen] - lead author to be decided later.

[10] F. Coccini, T. H. Upton, and W. Andreoni, J. Chem. Phys. 88, 6068 (1988).

[11] J. H. Van Vleck, J. Chem. Phys. 7, 72 (1939).

[12] C. A. Mead, Rev. Mod. Phys. 64, 51 (1992) uses a different set of variables $(Q, s, \theta)$ related to our variables by $Q=9\left[\left(r_{0}+Q_{1}\right)^{2}+Q^{2}+Q^{3}\right], Q s=18\left(r_{0}+Q_{1}\right) \sqrt{Q_{2}^{2}+Q_{3}^{2}}$, and $\theta=\tan ^{-1}\left(Q_{3} / Q_{2}\right)=\alpha$.

[13] The amplitudes $\left(Q_{1}, Q_{2}, Q_{3}\right)$ used here are smaller by $1 / \sqrt{3}$ than the conventional normal mode amplitudes, because we define them in terms of vectors $(\vec{\xi}, \vec{v})$ normalized to 1 on each atom, larger by $\sqrt{3}$ than is conventional.

[14] G. Herzberg and H. C. Longuet-Higgins, Disc. Faraday
Soc. 35, 77 (1963).

[15] For reprints of original papers see A. Shapere and F. Wilczek, Geometric phases in physics, World Scientific (1989).

[16] H. von Busch, V. Dev, H.-A. Eckel, S. Kasahara, J. Wang, W. Demtröder, P. Sebald, and W. Meyer, Phys. Rev. Lett. 81, 4584 (1998).

[17] G. Delacrétaz, E. R. Grant, R. L. Whetten, L. Wöste, and J. W. Zwanziger, Phys. Rev. Letters 56, 25989 (1986).

[18] To second order in $Q$ the truncated electron theory used here also predicts a warping of the adiabatic surface by $\left(g^{2} Q^{2} / 4 t\right) \cos (3 \alpha)$ which is minimized at the obtuse positions. This comes from off-diagonal coupling of the lower Jahn-Teller state $\left|\psi_{\text {ad }}\right\rangle$ to the state $|k=0\rangle$. This is not a reliable estimate since effects omitted in our theory will contribute similar corrections. See Sec. VI for further discussion.

[19] H. C. Longuet-Higgins, U. Öpik, M. H. L. Pryce, and R. A. Sack, Proc. Roy. Soc. A 244, 1 (1958).

[20] W. P. Su, J. R. Schrieffer, and A. J. Heeger, Phys. Rev. B 22, 2099 (1980).

[21] F. S. Ham, Phys. Rev. Letters 58, 725 (1987).

[22] M. D. Deshpande, D. G. Kanhere, I. Vasiliev, and R. M. Martin, Phys. Rev. A 65, 033202 (2002).

[23] J. Von Neumann and E. Wigner, Phys. Z. 30, 467 (1929).

[24] D. R. Yarkony, Rev. Mod. Phys. 68, 985 (1996).

[25] A. Barone, T. Hakioğlu, and I. O. Kulik, Eur. Phys. J. B 30, 219 (2002).

[26] T. Baruah, unpublished. 